\title{
An Efficient Approach for Uncertainty Analysis in Multidisciplinary Design Optimization
}

\author{
Laixi Zhang ${ }^{1, a}$, Yuan Zhang ${ }^{2}$ and Xiaolong Shen ${ }^{1, b}$ \\ ${ }^{1}$ Hunan Industry Polytechnic, Changsha, 410208, China \\ ${ }^{2}$ Hunan Sunrise Auto Mould \& Die Co., Ltd., Changsha, 410117, China \\ aIxzhzh@yahoo.com.cn, bShenXL64@163.com
}

Keywords: Multidisciplinary system design, Uncertainty, Response surface, Evidence theory.

\begin{abstract}
In this paper, an efficient multidisciplinary design optimization method based on evidence theory is proposed. Evidence theory is used to quantify uncertainty in terms of uncertainty measures of belief and plausibility. Since uncertainty measures provided by evidence theory are discontinuous functions, the response surface is utilized to obtain smooth functions so that the traditional gradient-based algorithms can be used in optimization.
\end{abstract}

\section{Introduction}

In general, probability theory is very effective when sufficient data about uncertainty are available to precisely construct probability distributions. However, when sufficient data are not available or there is lack of information due to ignorance, the classical probability theory may not be suitable. For example, the reliability of a complex system is assessed in the presence of incomplete information on the variability of certain design variables, parameters, operating conditions, boundary conditions, etc. A similar problem is: when quantification of a product's reliability or compliance to performance targets, it is practically very difficult due to insufficient data for modeling uncertainties during the early stages of product development.

Many of these new representations of uncertainty are able to more accurately represent epistemic uncertainty than traditional probability theory. Engineering applications of some of these theories can be found in recent publications [1,2]. One of the modern theories of uncertainty representation is evidence theory (Dempster-Shafer theory). The advantage of using evidence theory lies in the fact that it can be successfully used to quantify the degree of uncertainty when the amount of information available is small. Like most modern uncertainty theories, evidence theory also provides two uncertain measures known as belief and plausibility. In this paper, a formulation of evidence-based multidisciplinary optimization and design (EBMDO) is proposed for engineering design optimization.

\section{Uncertainty in Engineering Systems}

Uncertainty in single-discipline engineering systems. The sources of uncertainty in single discipline engineering systems lay the foundation in determining uncertainty of output states of multidisciplinary systems (see Fig. 1). There are three ways that uncertainties are introduced to the engineering systems because numerical models of engineering systems, such as finite element analysis (FEA) or computational fluid dynamics (CFD), are extensively used by the designers [3]. First, there is epistemic uncertainty when a physical model is converted into a mathematical model. That is because all the nonlinearity of the physical model cannot be exactly transformed into mathematical equations. Second, there is uncertainty in the data that are inputs to the system. Third, the mathematical equations can be solved using a variety of techniques and these different methods usually provide slightly different results.

Uncertainty in multidisciplinary systems. Multidisciplinary systems are complex engineering systems which can be inherently coupled. Under a multidisciplinary design environment, a system is 
composed of subsystems (disciplines). Each of these multidisciplinary subsystems uses a variety of disciplinary models with uncertainties associated with performance predictions. A schematic representation of a multidisciplinary system analysis is shown in Fig. 2. The system analysis is decomposed into three disciplines or contributing analyses (CAs). Each discipline or CA makes use of a simulation based discipline design tool. These disciplines are often highly coupled where the performance prediction of one discipline may become the input of another discipline and vice versa. The final output from the integrated multidisciplinary system has an accumulated effect of the uncertainties from the individual disciplines.

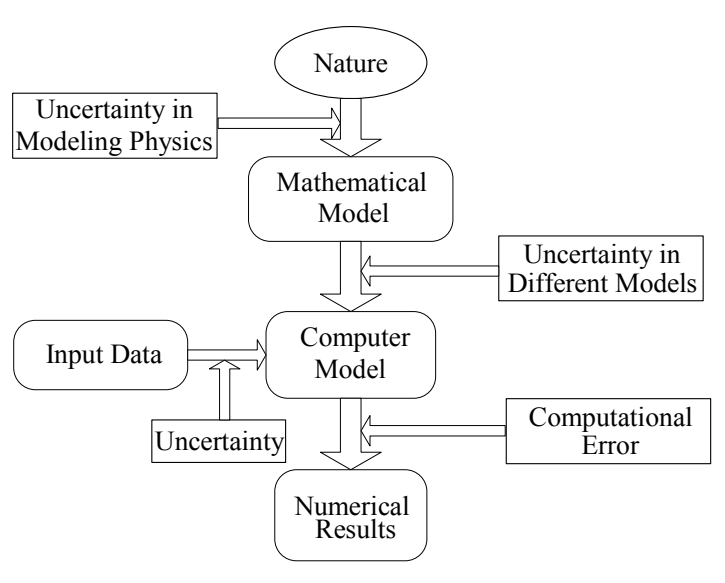

Fig. 1 Sources of uncertainty

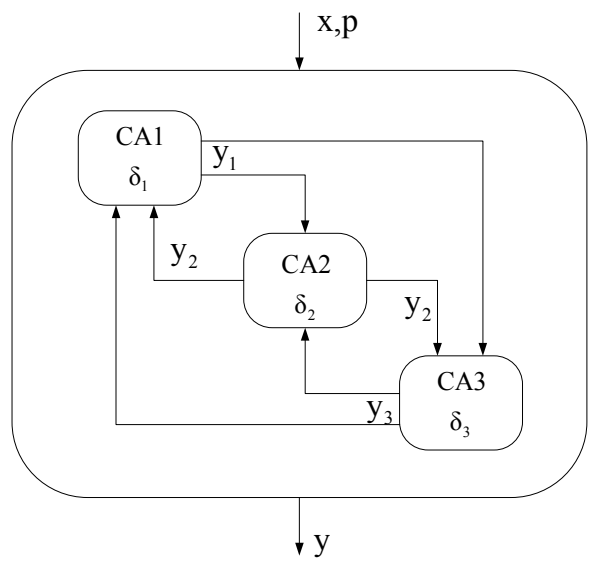

Fig. 2 Uncertainty in multidisciplinary system analysis

\section{Uncertainty in Engineering Systems}

In this section, the fundamentals of evidence theory and its way used in multidisciplinary design optimization are given.

Evidence theory is based on two measures of uncertainty, belief (Bel) and plausibility $(\mathrm{Pl})$. Belief and plausibility measures are determined from the known evidence for a proposition and it is not necessary to distribute the evidence to subsets of the proposition. So that evidence in the form of experimental data or expert opinion can be obtained for a parameter value within an interval. It is not necessary to assume a particular value within the interval or the likelihood of any value in the interval. The evidential measure for the occurrence of an event and the evidential measure for its negation do not have to sum to unity because of the existence of uncertainty. Once a body of evidence is given, belief and plausibility measures can be obtained by using the following formulas.

Sometimes the available evidence can come from different sources, and such bodies of evidences must be combined. If the BPAs $m_{1}$ and $m_{2}$ express evidence from two experts, the combined evidence $m$ can be calculated by the following formula.

$$
m(A)=\frac{\sum_{B \cap C=A} m_{1}(B) m_{2}(C)}{1-\sum_{B \cap C=\varnothing} m_{1}(B) m_{2}(C)} \quad, \quad A \neq \varnothing
$$

\section{Evidence Based Multidisciplinary Design Optimization (EBMDO)}

In deterministic Multidisciplinary Design Optimization (MDO), the design variables, parameters and responses are dealt with quantities that can be defined precisely. An objective function is minimized subject to satisfying each constraint. A conventional deterministic MDO problem is in the following form (containing two disciplinary).

where $D_{i}(i=1,2)$ is the vector of deterministic design variables, $D_{s}$ is the vector of deterministic sharing design variables, $P_{i}(i=1,2)$ is the vector of deterministic parameters, $Z_{i}(i=1,2)$ is the vector 
of output of discipline $i, Z_{i}^{c}(i=1,2)$ is the vector of coupling variables. $f$ is the objective function, and $g$ is the constraint.

$$
\begin{array}{ll}
\text { Find } & \multicolumn{1}{c}{D_{1}, D_{2}, D_{s}} \\
\text { To minimize } & f\left(D_{1}, D_{2}, D_{s}, P_{1}, P_{2}, Z_{1}, Z_{2}\right) \\
\text { Subject to } & g\left(D_{1}, D_{2}, D_{s}, P_{1}, P_{2}, Z_{1}, Z_{2}\right) \leq 0 \\
& Z_{1}=h_{1}\left(D_{1}, D_{s}, P_{1}, Z_{2}^{c}\right) \\
& Z_{2}=h_{2}\left(D_{2}, D_{s}, P_{2}, Z_{1}^{c}\right)
\end{array}
$$

In EBMDO, design variables are associated with uncertainty. The objective function is usually minimized subject to constraints that the required value of uncertain measure of satisfying each constraint is greater than a specified level. An EBMDO problem can be formulated as

Find $\quad D_{1}, D_{2}, D_{s}, X_{1}, X_{2}, X_{s}$

To minimize $f\left(D_{1}, D_{2}, D_{s}, X_{1}, X_{2}, X_{s}, P_{1}, P_{2}, P_{1}^{U}, P_{2}^{U}, Z_{1}, Z_{2}\right)$

$$
\begin{gathered}
\text { Subject to } U_{\text {reqd }}^{M}-U^{M}\left(g\left(\begin{array}{l}
D_{1}, D_{2}, D_{s}, X_{1}, X_{2}, X_{s} \\
, P_{1}, P_{2}, P_{1}^{U}, P_{2}^{U}, Z_{1}, Z_{2}
\end{array}\right) \leq 0\right) \leq 0 \\
Z_{1}=h_{1}\left(D_{1}, D_{s}, X_{1}, X_{s}, P_{1}, P_{1}^{U}, Z_{2}^{c}\right) \\
Z_{2}=h_{2}\left(D_{2}, D_{s}, X_{2}, X_{s}, P_{2}, P_{2}^{U}, Z_{1}^{c}\right)
\end{gathered}
$$

where $D_{i}(i=1,2)$ is the vector of deterministic design variables, and $D_{s}$ is the vector of deterministic design variables. $X_{i}(i=1,2)$ is the vector of design variables with uncertainty, and $X_{s}$ is the vector of sharing design variables with uncertainty. $P_{i}(i=1,2)$ is the vector of deterministic design parameters, $P_{i}^{U}(i=1,2)$ is the vector of design parameters with uncertainty. $Z_{i}(i=1,2)$ is the vector of output of discipline $i, Z_{i}^{c}$ is the vector of coupling variables. $f$ is the objective function. $U^{M}$ is the uncertain measure of constraint $g \leq 0$ that can be Bel or $\mathrm{Pl}$, and $U_{\text {reqd }}^{M}$ is the minimum required value of the uncertain measure.

Since constraints in Eq. (3) are discontinuous, in this paper the response surface approximations of each active constraint are used to obtain smooth functions. For establishing the response surface, the cross-validated moving least squares method is used based on an optimum symmetric latin hypercube space-filling sampling [4-5]. To calculate the uncertain measure of each active constraint, the focal elements partitioning method is used [6].

\section{Mathematical Example}

There are two design variables and two output states in the system analysis, the deterministic form of the optimization problem is as follows.

$$
\begin{aligned}
& \text { Find } \\
& \text { To minimize } \\
& \begin{array}{lc} 
& x_{1}, x_{2} \\
\text { Subject to } & g_{1}=y_{1}-8 \geq 0 \\
& g_{2}=5-y_{2} \geq 0 \\
& -10 \leq x_{1} \leq 10 \\
& 0 \leq x_{2} \leq 10
\end{array}
\end{aligned}
$$

where the states $y_{1}$ and $y_{2}$ are calculated by CA1 and CA2 respectively, as

$$
\begin{aligned}
& C A_{1}: y_{1}=x_{1}^{2}+x_{2}-0.2 y_{2} \\
& C A_{2}: y_{2}=x_{1}-x_{2}^{2}+\sqrt{y_{1}}
\end{aligned}
$$


The deterministic form of the mathematical example is modified to fit the optimization under uncertainty using evidence theory. The uncertainty is denoted by $\delta_{1}$ and $\delta_{2}$ in CA1 and CA2 respectively. The information on the values of the epistemic uncertainty for $\delta_{1}$ and $\delta_{2}$ is obtained through expert opinion. Intervals for the uncertainty and the corresponding BPA are provided in Table 1. When the uncertainty is considered, the EBMDO problem is formulated as

$$
\begin{array}{lc}
\text { Find } & x_{1}, x_{2} \\
\text { To minimize } & f(\mathrm{x})=x_{1}^{2}+10 x_{2}^{2}+y_{1} \\
\text { Subject to } & G_{1}=U^{M}\left(y_{1}-8 \geq 0\right)-U_{\text {reqd }}^{M} \geq 0 \\
& G_{2}=U^{M}\left(5-y_{2} \geq 0\right)-U_{\text {reqd }}^{M} \geq 0 \\
& -10 \leq x_{1} \leq 10 \\
& 0 \leq x_{2} \leq 10
\end{array}
$$

The meaning of $U^{M}$ and $U_{\text {reqd }}^{M}$ has been mentioned above and here the uncertain measure used is

\begin{tabular}{|c|c|c|c|c|c|c|c|}
\hline \multicolumn{4}{|c|}{$\delta_{1}$} & \multicolumn{4}{|c|}{$\delta_{2}$} \\
\hline expert & & expert & & expert 1 & & expert & \\
\hline Interval & BPA & Interval & BPA & Interval & BPA & Interval & $\mathrm{BP}$ \\
\hline$\left[\begin{array}{ll}-1 & -0.5\end{array}\right]$ & 0.2 & {$\left[\begin{array}{ll}-0.8 & -0.5\end{array}\right]$} & 0.1 & {$\left[\begin{array}{lll}-0.75 & -0.25\end{array}\right]$} & 0.2 & {$\left[\begin{array}{lll}-0.7 & 0.1\end{array}\right]$} & 0 . \\
\hline$\left[\begin{array}{lll}-0.5 & 0.5\end{array}\right]$ & 0.5 & {$\left[\begin{array}{lll}-0.5 & 0.5\end{array}\right]$} & 0.7 & {$\left[\begin{array}{lll}-0.25 & 0.5\end{array}\right]$} & 0.5 & {$\left[\begin{array}{ll}0.1 & 0.75\end{array}\right]$} & 0.4 \\
\hline$\left[\begin{array}{ll}0.5 & 1\end{array}\right]$ & 0.3 & {$\left[\begin{array}{ll}0.5 & 0.6\end{array}\right]$} & 0.2 & {$\left[\begin{array}{ll}0.5 & 0.7\end{array}\right]$} & 0.3 & - & - \\
\hline
\end{tabular}
Bel. The minimum required value of belief is taken to be 0.99 . Table 2 summarizes and compares results of deterministic MDO and EBMDO. As expected, the deterministic optimum of 15.98 is less than the EBMDO optimum of 17.64. The EBMDO design is more conservative compared with the deterministic MDO for a more reliable design.

Table 1. BPA structure for $\delta_{1}$ and $\delta_{2}$
Table 2. Comparison of design results of mathematical example

\begin{tabular}{cccc}
\hline & Starting point & MDO & EBMDO \\
\hline$x_{1}$ & -5 & -2.82 & -2.96 \\
$x_{2}$ & 3 & 0.05 & 0.06 \\
$f$ & 94.7 & 15.98 & 17.64 \\
$y_{1}$ & 29.7 & 8 & 8.8 \\
$y_{2}$ & -8.5 & 0.006 & 0.019 \\
\hline
\end{tabular}

\section{Summary}

In this paper, an approach for performing optimization under uncertainty is presented. Evidence theory was used to assess design reliability with incomplete information. Uncertainties of constraints posed in the design optimization problem are quantified using expert opinions. The belief measure is used in this paper to evaluate non-deterministic constraints. Since the belief functions are discontinuous, a local response surface is generated. Examples are used to demonstrate the proposed EBMDO method. It was shown that the EBMDO design is more conservative than the deterministic MDO.

\section{Acknowledgements}

This project is supported by Hunan Industry Polytechnic Grant (No. GYKYZ2012017).

\section{References}

[1] Z.P. Mourelatos, J. Zhou. A design optimization method using evidence theory. Journal of Mechanical Design, 128(2006), p. 901-908.

[2] J.F.G. Oliveira, T.V. Franca and J.P. Wang: CIRP Ann. Vol. 57 (2008), p. 329-332.

[3] X.L. Shen, L.X. Zhang, H.Long and Z.X. Zhou: Appl. Mech. Mater. Vol. 34-35 (2010), p. 1936-1940.

[4] R.J. Ong, J.T. Dawley and P.G. Clem: submitted to Journal of Materials Research (2003)

[5] X.L. Shen, Y.X. Luo, L.X. Zhang and H. Long: Adv. Mater. Res. Vol. 156-157(2011), p. $1582-1585$.

[6] K.Q. Ye, W. Li and A. Sudjianto. Algorithmic construction of optimal symmetric Latin hypercube designs. Journal of Statistical Planning and Inference, 90(2000), p. 145-159. 